Finanz. polit. econ., ISSN: 2248-6046, Vol. 11, N. ${ }^{\circ} 2$, julio-diciembre, 2019, pp. 353-374

http://doi.org/10.14718/revfinanzpolitecon.2019.11.2.8
Raúl de Jesús-Gutiérrez*

Recibido: 23 de enero de 2018

Concepto de evaluación: 23 de marzo de 2019

Aprobado: 12 de diciembre de 2019

Artículo de investigación

(c) 2019 Universidad Católica de Colombia.

Facultad de Ciencias

Económicas y Administrativas.

Todos los derechos reservados

\section{Integración entre mercados de petróleo de diferente calidad con base en las correlaciones condicionales dinámicas ${ }^{1}$}

\section{RESUMEN}

Este artículo prueba el grado de integración de México en los mercados internacionales del petróleo a través de la evolución de las correlaciones dinámicas en periodos estables, de crisis e inestables. Las estimaciones del modelo GARCH-CCD muestran que las correlaciones son positivas y cambian con el tiempo en respuesta al origen de choques en los precios del petróleo para los periodos de relativa calma, crisis y turbulencia financiera. Asimismo, los resultados del estadístico-t y valor-p bootstrap confirman que las correlaciones son significativamente diferentes en periodos de estabilidad e inestabilidad con respecto a las correlaciones del periodo de crisis, lo que favorece la hipótesis de regionalización entre los mercados de petróleo. Los hallazgos tienen importantes implicaciones económicas y financieras para el gobierno y los consumidores.

Palabras clave: correlaciones condicionales dinámicas, crisis financieras, integración de los mercados de petróleo.

JEL Classification: C22, F20, F41, Q40

Cómo citar este artículo / To reference this article / Para citar este artigo:

de Jesús-Gutiérrez, R. (2019). Integración entre mercados de petróleo de diferente calidad con base en las correlaciones condicionales dinámicas. Revista Finanzas y Política Económica, 11(2), 353-374. doi: http://dx.doi.org/10.14718/ revfinanzpolitecon.2019.11.2.8

Integration among world and low quality crude oil markets based on dynamic conditional correlations

\section{ABSTRACT}

This paper tests the degree of integration between Mexico's and world crude oil markets throughout the evolution of dynamics correlations during the stable, crisis and volatile periods. The estimations of DCC-GARCH model show that the correlations are positive and time-varying in responds to the

\footnotetext{
de tiempo completo de la Facultad de Economía de la Universidad Autónoma del Estado de México. Correo electrónico:
rjg2005mx@yahoo.com.mx. del Estado de México. Correo electrónico:
rjg2005mx@yahoo.com.mx. (D) https://orcid.org/0000-0001-6878-3038

* Doctor en Ingeniería Financiera; profesor
}
1 Este artículo se deriva de un proceso de investigación adelantado en la Facultad de Economía de la Universidad Autónoma del Estado de México.


origin of the oil price shocks in periods of relative calm and financial turmoil. Likewise, the results of statistic-t and bootstrap $p$-value confirm strongly that the correlations in the crisis period are significantly different from those in the stable and volatile periods, which provides evidence in favor of the regionalization hypothesis between crude oil markets. The findings have important economic and financial implications for the government and consumers.

Keywords: Dynamics conditional correlations, financial crises, integration crude oil markets.

\section{Integração entre mercados de petróleo de diferente qualidade com base nas correlações condicionais dinâmicas}

\section{RESUMO}

Este artigo testa o grau de integração do México nos mercados internacionais do petróleo por meio da evolução das correlações dinâmicas em períodos estáveis, de crise e instáveis. As estimativas do modelo GARCH-CCD mostram que as correlações são positivas e se transformam com o tempo em resposta à origem de choques nos preços do petróleo para os períodos de relativa calma, crise e turbulência financeira. Além disso, os resultados do estatístico-t e valor-p bootstrap confirmam que as correlações são significativamente diferentes em períodos de estabilidade e instabilidade a respeito das correlações do período de crise, o que favorece a hipótese de regionalização entre os mercados de petróleo. Os achados têm importantes consequências econômicas e financeiras para o governo e para os consumidores.

Palavras-chave: correlações condicionais dinâmicas, crises financeiras, integração dos mercados de petróleo. 


\section{INTRODUCCIÓN}

La integración o regionalización de los mercados internacionales de petróleo ha despertado recientemente gran interés en los académicos, diseñadores de política económica y energética, gobiernos, inversionistas institucionales y reguladores. De hecho, el estudio de la sincronización dinámica entre los principales mercados de energía del mundo tiene importantes implicaciones económicas para la política energética y el crecimiento económico de varios países. De igual manera, el mejoramiento de la política de competencia en los mercados de energía de baja calidad puede incrementar los ingresos de los países exportadores de crudo, lo que contribuye a incrementar los recursos para el desarrollo de proyectos sociales de sus habitantes - p. e., seguridad social y educación-, particularmente en economías emergentes.

De acuerdo con Energy Information Administration (2012), el 17,5\% de la producción global está constituida por tres principales marcadores: a) West Texas Intermediate de Estados Unidos (WTI, por sus siglas en inglés), b) Brent de Londres, del Mar del Norte, y c) Dubái de Medio Oriente; todos ellos considerados, por su alta densidad, los crudos más reconocidos y negociados en el ámbito mundial ${ }^{1}$. En general, los crudos WTI y Brent comparten casi las mismas características de acuerdo con la medida internacional del Instituto Americano del Petróleo (API, por sus siglas en inglés). Además, su comercialización se lleva a cabo en mercados de contado y futuros altamente líquidos con bajos costos de transacción que contribuyen rápidamente al ajuste de los precios para evitar oportunidades de arbitraje. Sin embargo, la vasta variedad de petróleos extraídos de diferentes zonas geográficas del mundo, aunada a la creciente

1 Desde la segunda mitad de los ochenta, el Dubái ha sido el principal marcador de referencia para el petróleo pesado de la región del Golfo Pérsico. Pese a ser un crudo ácido de densidad media, su cotización es clave en el proceso de la fijación de precios de los crudos de Arabia Saudita, Emiratos Árabes Unidos, Irán, Iraq y Kuwait (todos ellos, miembros de la OPEP). Además, este tipo de petróleo se negocia en las bolsas de Singapur (SIMEX) y Nueva York (NYMEX). comercialización de diversos niveles de calidad, ha impulsado el continuo crecimiento en los mercados de energía fósil, al tiempo que se incrementa la volatilidad en su estructura como consecuencia de choques exógenos, que incluyen las crisis financieras, los conflictos geopolíticos y los cambios climáticos (incluso los de carácter especulativo).

En la actualidad, el $50 \%$ de la producción global aproximadamente está constituida por nuevos tipos de petróleo de calidad media-pesada y distintas características de procesamiento (Montepeque, 2005). El fácil acceso a múltiples mercados líquidos y no líquidos ha contribuido a la evolución en forma paralela de los precios de los diferentes tipos de petróleo, lo que da pauta a la discusión de las características de integración y regionalización de los mercados de petróleo desde la perspectiva de la relación estructural interdependiente de los precios del petróleo en un contexto internacional. A este respecto, Adelman (1984) argumenta que el mercado internacional del petróleo, como el océano del mundo, es un gran estanque sin dimensiones. Esta hipótesis confirma la existencia de un mercado global altamente integrado para los diferentes mercados de petróleo, puesto que los cambios en las condiciones mercantiles de una región pueden tener repercusiones en el comportamiento de otros mercados regionales.

En contraste, Weiner (1991) sustenta que los mercados de petróleo se encuentran altamente regionalizados debido a que los movimientos en sus precios presentan una tendencia independiente como respuesta a las políticas gubernamentales domésticas y a los choques regionales. En la literatura, estas dos hipótesis han sido discutidas profundamente a través de diferentes técnicas econométricas que utilizan datos de diversa frecuencia y periodos de análisis, lo que ha conducido a evidencia empírica mixta sobre la hipótesis de integración o regionalización. El hecho en mención puede ser explicado por los escenarios de volatilidad y los fuertes comovimientos que ha experimentado la estructura de los mercados internacionales de petróleo a través del tiempo, obviamente influenciados por las heterogéneas características de calidad y la interacción 
de los precios de la amplia variedad de tipos de petróleos comercializados en mercados regionales.

En consecuencia, el objetivo del presente trabajo es analizar si el mercado mexicano del petróleo se encuentra plenamente integrado o regionalizado con los mercados de petróleo de referencia internacional. En el análisis se pretende contestar la siguiente pregunta: ¿el análisis de las correlaciones dinámicas en diferentes periodos proporciona suficiente información acerca de las características de integración o regionalización entre mercados de petróleo de distinta calidad? En este contexto, el concepto de integración entre mercados de petróleo es explicado cuando los precios de los crudos están altamente correlacionados y el comportamiento dinámico de las correlaciones presenta la misma intensidad, independientemente de si las condiciones de mercado se encuentren en un periodo de estabilidad, crisis o inestabilidad.

Existen varias razones para estudiar la hipótesis de integración entre los mercados internacionales de petróleo y el mercado mexicano del petróleo. En primer lugar, México como productor de petróleo se ubicó en el undécimo lugar a nivel mundial en el 2016, con 2154 miles de barriles diarios en promedio, que equivalen aproximadamente al 3,32\% de la producción global, a pesar de no pertenecer a la Organización de Países Exportadores de Petróleo (OPEP). Así, sus exportaciones registraron 1194 miles de barriles diarios, con un valor de \$15.575 millones de dólares, donde el 59,59\% se destinaron a los Estados Unidos de América, su principal importador de energía fósil, seguido por Europa y Asia, con 22,78\% y 23,63\%, respectivamente. En segundo lugar, con la creciente expansión y diversificación de la comercialización del petróleo nacional, y con la apertura de capital privado nacional y extranjero para la exploración y extracción de petróleo en aguas profundas, México podría convertirse en un activo e importante proveedor para garantizar el hidrocarburo en el mundo y, en consecuencia, los precios del petróleo mexicano podrían llegar a influir en los precios internacionales del mercado, lo que le permitiría al país adoptar un nuevo sistema de fijación de precios. En tercer lugar, la mayoría de la literatura previa trata el tema de la integración-regionalización de los mercados de petróleo utilizando los precios de referencia internacional del WTI, Brent y Dubái; por ello, hasta el momento no existe un estudio sobre la integración entre el mercado mexicano petrolero y los mercados internacionales de petróleo con base en las correlaciones dinámicas entre dos series de rendimientos de los precios del petróleo en diferentes periodos.

En el presente artículo, en función de aportar a la literatura sobre la hipótesis de integración o regionalización en los mercados del petróleo, el modelo GARCH de correlación condicional dinámico es empleado para describir la estructura dinámica de las correlaciones entre los mercados internacionales y nacionales de petróleo. La principal ventaja del modelo se atribuye a que puede detectar cambios en las correlaciones a través del tiempo, lo que proporciona información más completa del fenómeno de las interrelaciones de los precios del petróleo en periodos de relativa calma, crisis financieras e inestabilidad, a diferencia de la medida convencional de Pearson utilizada por Weiner (1991). Sin embargo, el modelo no captura totalmente la no-linealidad observada en las series financieras, como el método de cópulas.

Ahora bien, el análisis de la estructura de dependencia es estática, por lo que también requiere de la filtración de los rendimientos a través de modelos GARCH para obtener una estructura de dependencia condicional. De esta manera, el análisis fuera de la muestra del comportamiento de las correlaciones condicionales dinámicas (de aquí en adelante, CCD) es importante porque los mercados reaccionan de forma instantánea a la llegada de nueva información, la cual afecta las varianzas y, por ende, el patrón de las CCD en diferentes niveles. En consecuencia, su omisión puede proporcionar resultados sesgados en la explicación de la hipótesis de integración o regionalización de los mercados del petróleo. 
Jia et al. (2015) sustentan que el problema de las fluctuaciones en los precios del petróleo es el resultado de la interacción de múltiples factores, dadas las diversas características. En este sentido, las correlaciones pueden ser de mayor utilidad para capturar las diferencias en los cambios de los precios. Asimismo, la naturaleza cambiante en el tiempo de las correlaciones - similar al de un proceso no estacionario- proporciona más información para explicar la hipótesis de integración o regionalización que las características de las fluctuaciones de los precios del petróleo, que comúnmente son utilizadas en el análisis de cointegración y modelo de vectores de corrección de error. Finalmente, el estudio usa la prueba $t$ basada en un análisis bootstrap para validar la consistencia de las CCD en los periodos de relativa calma e inestabilidad, contra las CCD del periodo de crisis. Este ejercicio es fundamental porque evidencia empírica ha demostrado que la intensidad de las CCD tiende a cambiar dramáticamente en los periodos de movimientos de precios extremos (Lanza, Manera y McAleer, 2006).

El artículo consta de seis secciones: la presente introducción, la revisión de la literatura relacionada, una descripción y análisis preliminar de los datos, la explicación de la metodología econométrica utilizada para probar la existencia de integración entre los mercados de petróleo, la discusión de los principales resultados y, finalmente, las conclusiones.

\section{LITERATURA RELACIONADA}

Como se mencionó, la literatura sobre el debate de la integración o regionalización de los mercados internacionales del petróleo es amplia y ha sido documentada desde varias técnicas econométricas y frecuencias de datos, pero con evidencia empírica mixta. Analizando los resultados de las correlaciones lineales y la regresión con variables de estado a través del ajuste de precios por regiones, Weiner (1991) encuentra evidencia empírica de regionalización entre los mercados de petróleo en la medida en que los precios de los hidrocarburos de la misma calidad se desvían unos de otros. En contraste, aplicando técnicas de cointegración a datos semanales y mensuales de los precios del petróleo de alta calidad, Gülen $(1997,1999)$ muestra evidencia consistente de integración en el corto plazo entre los mercados de petróleo.

Entre los estudios que confirman la hipótesis de que los mercados globales de petróleo están integrados, se puede mencionar el de Ewing y Harter (2000), que, estimando un modelo de vectores de corrección de error para datos mensuales en el periodo 1974-1996, muestran que los precios del Brent y North Slope de Alaska siguen una caminata aleatoria y mantienen una tendencia común en el largo plazo. Por su parte, Kleit (2001) mejora y extiende la aproximación de costo de arbitraje para datos semanales de siete mercados de petróleo de alta calidad; sus hallazgos revelan que los costos de transacción en los mercados internacionales de petróleo se reducen con el tiempo, y que la mayoría de los mercados estudiados están cada vez más interrelacionados. En contraste, Milonas Henker (2001) analizan la dinámica de los diferenciales de los precios del WTI y Brent, tras lo cual encuentran que los dos mercados de petróleo no están plenamente integrados. Utilizando datos de precios diarios de cinco petróleos de diferentes regiones geográficas, Bachmeier y Griffin (2006) estiman un modelo de corrección de error y llegan a la conclusión de que el mercado global del petróleo es único y altamente integrado en un contexto económico. De igual manera, Bentzen (2007) rechaza la hipótesis de regionalización y favorece el supuesto de mercado integrado empleando datos de frecuencia alta de los petróleos de la canasta de la OPEP, WTI y Brent, para el periodo 1988-2004.

Más tarde, aplicando cointegración asimétrica a los diferenciales de los precios de cuatro marcadores del petróleo (WTI, Brent, Dubái y Maya), Hammoudeh, Thompson y Ewing (2008) proporcionan evidencia de asimetría en el proceso de ajuste de los precios en el largo plazo, lo que confirma la globalización o integración de los mercados de petróleo. Desde esta perspectiva, y a 
partir de un proceso autorregresivo de dos regímenes, Fattouh (2010) señala que las series de los diferenciales en los precios del petróleo tienen una raíz unitaria, pero no son estacionarias (reversión a la media) en algunos periodos, lo que implica que los mercados de petróleo no necesariamente están perfectamente integrados en el corto plazo.

Reboredo (2011) analiza la estructura dependiente de una variedad de petróleos de diferente calidad mediante la técnica de cópulas. Sus hallazgos empíricos proporcionan evidencia en favor de la hipótesis de globalización o integración, debido a que los precios tienden a moverse en forma conjunta y con la misma intensidad, independientemente de las condiciones del mercado (normal o inestabilidad). Aplicando medidas de correlación no lineales y un modelo de vectores de corrección de error bajo dos regímenes, Liu, Chen y Wan (2013) contradicen los hallazgos de Li y Leung (2011), en cuyo estudio sustentan firmemente el hecho de que el mercado petrolero chino se encuentra perfectamente integrado a los mercados internacionales en la actualidad. Para los mercados del petróleo de Estados Unidos y Canadá, Wilmot (2013) argumenta que la hipótesis de integración requiere de la existencia de la relación de largo plazo entre petróleos que no son de referencia internacional. Los resultados del análisis de cointegración confirman la relación de largo plazo, pero débil por la presencia de un cambio estructural en el vector de cointegración, el cual coincide con la crisis financiera global.

Recientemente, Liao, Lin y Huang (2014) usaron pruebas de raíces unitarias basadas en los cuantiles con cambios estructurales para detectar que el comportamiento dinámico de los diferenciales de los precios de los crudos sigue un proceso estacionario en los cuantiles superiores. Sus resultados son robustos y apoyan la hipótesis de globalización en los mercados internacionales de petróleo para datos de diferentes frecuencias. En un análisis restringido a los petróleos de referencia internacional WTI, Brent, Dubái y Tapis, Lu et al. (2014) encontraron que los mercados del petróleo están integrados en periodos de inestabilidad financiera. Usando un modelo VEC en combinación con una técnica gráfica acíclica, Ji y Fan (2015) encuentran evidencia de que los mercados de petróleo WTI, Brent, Tapis y Nigeria están fuertemente integrados en el periodo 2000-2010. No obstante, la hipótesis de integración se vuelve débil después de que eventos atípicos tienen lugar al final del 2010. En estudios más recientes, Ruiz y Anguiano (2016) modelan la dinámica de las volatilidades e interrelaciones de los rendimientos de la mezcla mexicana de petróleo y los crudos Brent y WTI a través de un modelo DBEKK-TGARCH $(1,1)$.

Kuck y Schweikert (2017) emplean un modelo VEC con cambios de régimen para analizar el comportamiento de los ajustes de los estados de desequilibrio de la relación de largo plazo en los precios del WTI, Brent, Boni ligero, Dubái y Tapis. Los hallazgos muestran que los mercados del petróleo están integrados, y su grado de integración está estrechamente vinculado a la incertidumbre de la economía global. En el contexto de las razones de cobertura óptima del petróleo mexicano, De Jesús (2016) proporciona evidencia de que las volatilidades y correlaciones entre mercados de contado y futuros presentan un comportamiento dinámico a lo largo del tiempo y son altamente persistentes. Por su parte, Domínguez, Venegas y Palafox (2018) revelan que existe una relación de largo plazo entre la mezcla mexicana de petróleo y los crudos de referencia internacional Brent y WTI.

\section{DESCRIPCIÓN Y ANÁLISIS PRELIMINAR DE LOS DATOS}

La composición del mercado internacional del petróleo está formada por una diversidad de crudos con propiedades físico-químicas heterogéneas, extraídos de diferentes regiones y comercializados en varios mercados del mundo. Algunos tipos de petróleo, por sus características básicas en cuanto al bajo porcentaje de contenido de azufre ${ }^{2} \mathrm{y}$ alto grado

\footnotetext{
2 Los petróleos con bajo contenido de azufre son clasificados como dulces, mientras que los petróleos con alto contenido de azufre son considerados ácidos, esto es, cuando exceden el $0,5 \%$.
} 
de densidad $\mathrm{API}^{3}$, son utilizados como referencia para la fijación de precios de otros tipos de petróleo de menor calidad y pesados ${ }^{4}$. Por consiguiente, sus precios se negocian con un descuento debido a sus altos costos de refinación y riesgo de contaminación al medioambiente. México como exportador de petróleo produce tres tipos:

- Maya: un crudo ácido-pesado con 3,3\% de azufre en peso y densidad API de 21,8 grados.

- Istmo: un crudo ácido-mediano con $1,3 \%$ de azufre en peso y densidad API de 33,4 grados.

- Olmeca: un crudo ácido-ligero con 0,8\% de azufre en peso y densidad API de 39,3 grados.

Debido a que los choques de la oferta y demanda afectan directamente los precios del petróleo de las principales zonas geográficas de producción, y a que sus efectos positivos o negativos son inmediatamente transmitidos a otros mercados locales, este trabajo utiliza los precios diarios del petróleo Maya, Istmo y Olmeca, y toma como referencia los precios diarios de los petróleos WTI y Brent para representar al mercado internacional del petróleo. El análisis cubre el periodo del 2 de enero de 1997 al 31 de diciembre del 2016, totalizando 5174 observaciones. Las series de los precios se obtuvieron de la base de datos Bloomberg. En el análisis es importante resaltar que la muestra total se divide en tres submuestras: 1997-2003, 2003-2009 y 2010-2016. En la estimación de las correlaciones fuera de la muestra se utiliza una ventana móvil, la cual consiste en remover la observación más remota y agregar la observación más reciente.

En la figura 1 se describe el comportamiento dinámico de los precios del petróleo nacional e internacional durante el periodo 1997-2016. El petróleo WTI alcanza valores por encima de los

$3 \quad$ Los petróleos con densidad API mayor de 35 grados son considerados ligeros, con densidad API entre 26 y 35 grados son medianos, en tanto que los crudos con API menores a 26 grados son conocidos como pesados.

4 Los petróleos de referencia WTI y Brent son dulces-ligeros, con densidades API de 40 y 38 grados y contenido de azufre de $0,2 \%$ y $0,4 \%$, respectivamente. otros tipos de petróleo, en especial el crudo Maya. Sin embargo, el crudo Olmeca compite en calidad y precio con los principales marcadores, particularmente durante el periodo 2010-2016, en el que notablemente se puede observar que sus cotizaciones tienen una prima adicional con respecto al WTI, aunque con un precio menor con respecto al Brent durante el mismo periodo. Además, la mayor parte de la demanda del Olmeca es generalmente empleada para transformar petróleos pesados en mezclas más ligeras que cumplan con los estándares de regulación ambiental. Asimismo, la dinámica de los precios da la impresión de que los mercados nacionales e internacionales de petróleo se mueven en una misma dirección, aunque con diferentes intensidades que dependen de las características de los tipos de crudo.

En el periodo 1997-1998, los precios del petróleo presentaron una tendencia a la baja como consecuencia de la tensión en Medio Oriente y la crisis asiática de 1997-1998. Igualmente, desde el atentado terrorista del 11 de septiembre del $2001 \mathrm{y}$ el conflicto militar entre Estados Unidos e Irak en el 2003, los precios físicos del petróleo nunca volvieron a alcanzar los niveles de 1998, a tal grado que en julio del 2008 se registraron precios históricos de $\$ 145,31$ y $\$ 146,38$ dólares por barril. Entre los factores que explican este hecho se pueden mencionar al exceso en la demanda del hidrocarburo por parte de economías emergentes (China y la India ${ }^{5}$ ), los recortes de la producción con la intención de regular precios y, quizás el más importante, el síntoma desmedido de la especulación entre el 2005 y el 2007. Finalmente, con la intensificación de la crisis financiera global y la restricción del crédito, justamente el 15 de julio del 2008 los precios del petróleo iniciaron una dramática tendencia a la baja hasta alcanzar niveles de entre 36,74 y 44,60 dólares a fines de diciembre.

5 De acuerdo con las estadísticas de energía mundial de British Petroleum (2017), China e India son importantes consumidores de energía fósil, con un volumen de aproximadamente 11.986 y 4.164 miles de barriles diarios en promedio, lo que representa el $49,70 \%$ de la región de Asia Pacífico y el $16,99 \%$ del consumo total. 
Figura 1.

Comportamiento de los precios del petróleo nacional e internacional
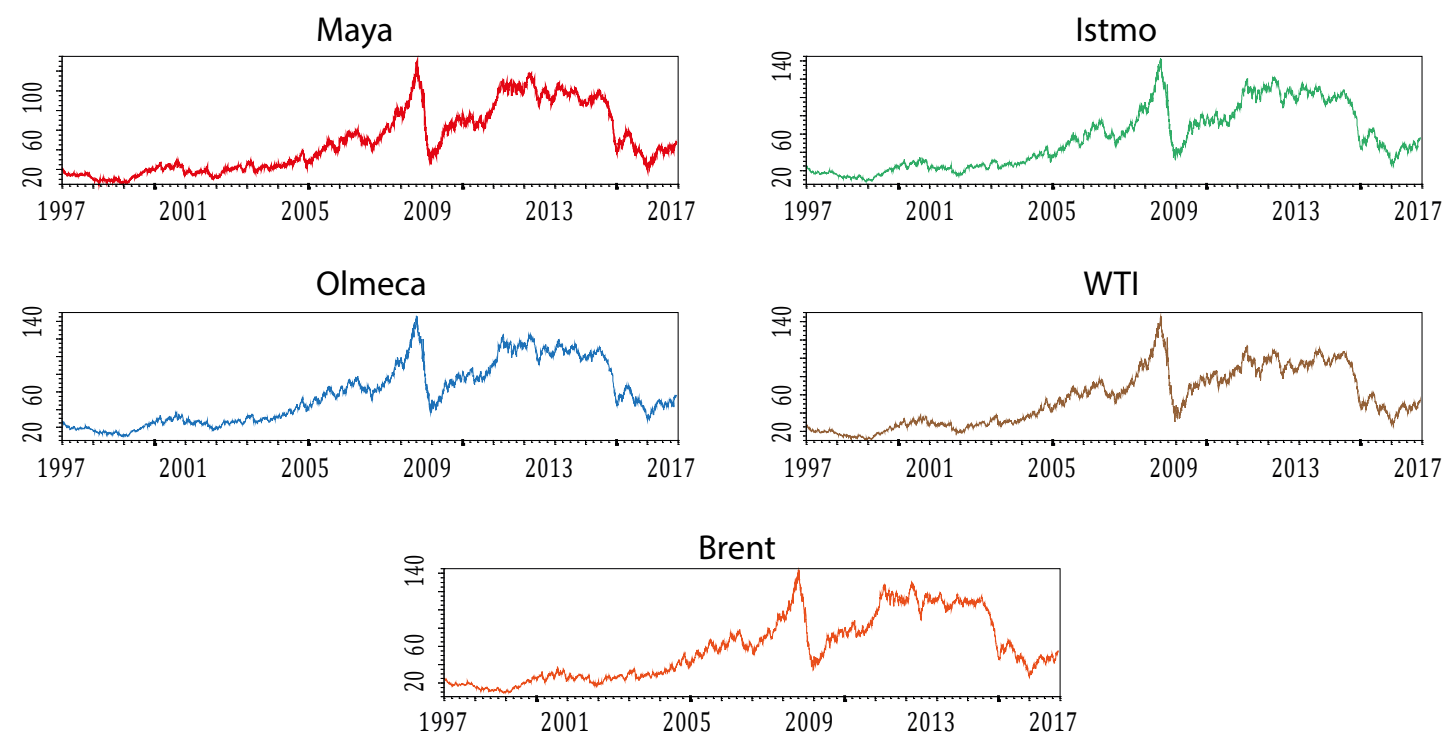

Fuente: elaboración propia con datos de Bloomberg.

No obstante, la recuperación económica de Estados Unidos de América, los ajustes en la producción de la OPEP, los disturbios en Libia y la inestabilidad política en Egipto fueron factores fundamentales para impulsar nuevamente los precios al alza y mantener una fluctuación en una banda de entre 75 y 110 dólares. Después de este importante repunte, los precios del petróleo nuevamente inician un episodio bajista en junio del 2014, el cual se prolonga hasta alcanzar el precio promedio de 25,99 dólares en febrero del 2016 y cerrar con un precio promedio de 53,65 dólares por barril, hecho principalmente alimentado por las frecuentes interrupciones del suministro y el control de la producción ejercido por la OPEP. Estos vaivenes inesperados en los precios hangenerado notables cambios estructurales en las correlaciones de los mercados del petróleo, en particular durante los periodos de crisis e inestabilidad económica.

Los precios del petróleo cotizan en dólares estadounidenses por barril. Todas las series son transformadas a procesos estacionarios a través de la diferencia entre el logaritmo del precio actual y el logaritmo del precio previo, es decir, $r_{t}=\ln \left(P_{t}\right)-\ln \left(P_{t-1}\right)$. El tamaño de la muestra permite el análisis de las series del petróleo en tres periodos: en 1997-2003 (antes de la crisis), en 2003-2009 (de crisis) y en 2010-2016 (de inestabilidad). La tabla 1 reporta las estadísticas básicas de los tres periodos de análisis. Los resultados indican claramente que las medias de los rendimientos son relativamente pequeñas. En contraste, las desviaciones estándar presentan valores altos en los tres periodos, hecho que implica que los mercados de petróleo son altamente volátiles y las correlaciones pueden cambiar en el tiempo.

Otra importante observación es que las medias de los rendimientos son considerablemente más altas en el periodo 2003-2009, de crisis, y negativas en el periodo 2010-2016, de inestabilidad, como consecuencia de la presencia de fluctuaciones extremas en intervalos cortos de tiempo. Las series de los rendimientos del petróleo presentan sesgo negativo en el periodo estable y positivo en el periodo de inestabilidad, mientras el coeficiente es mixto en el periodo de crisis. Asimismo, el exceso de curtosis es notable en las series de los rendimientos 
del petróleo para los tres periodos de estudio. Este hecho implica que la distribución de rendimientos presenta propiedades de colas más anchas y largas que las de la distribución normal. Además, el supuesto de normalidad de la distribución incondicional es fuertemente rechazado por la prueba Jarque-Bera (JB) en los diferentes periodos de análisis.

Los resultados de la prueba de Ljung-Box indican la presencia de correlación serial en los rendimientos cuadrados a un nivel de significancia de $1 \%$, así como una fuerte presencia de efectos $\mathrm{ARCH}$ en los rendimientos para los tres periodos.
Esta característica común en los rendimientos está ampliamente sustentada en la figura 2 , donde se puede observar fuerte evidencia de volatilidad en aglomeraciones o periodos de alta volatilidad, seguidos de periodos de relativa tranquilidad, en particular para el periodo de crisis. Estos hallazgos sugieren el uso de modelos GARCH multivariados para analizar el comportamiento de las volatilidades condicionales y correlaciones dinámicas entre los mercados del petróleo mexicano y los internacionales.

Las condiciones de estacionariedad en las series de los niveles de los precios y las primeras

Tabla 1.

Estadísticas básicas de los rendimientos para los diferentes tipos de petróleos

\begin{tabular}{|c|c|c|c|c|c|c|c|c|c|}
\hline & Media & Desviación & Máximo & Mínimo & Sesgo & Curtosis & JB & LM & $Q^{2}(20)$ \\
\hline \multicolumn{9}{|c|}{ Panel A: periodo estable 1997-2003 } & \\
\hline WTI & 0,0091 & 2,5451 & 14,8923 & $-16,5512$ & $-0,3152$ & 7,0074 & $1154^{*}$ & $52,91^{*}$ & $64,44^{*}$ \\
\hline Brent & 0,0106 & 2,6150 & 13,3735 & $-16,6312$ & $-0,1922$ & 6,5642 & $901^{*}$ & $51,97^{*}$ & $68,20^{*}$ \\
\hline Maya & 0,0013 & 2,9775 & 17,8723 & $-19,1634$ & $-0,1075$ & 8,0152 & $1767^{*}$ & $138,99^{*}$ & $321,26^{*}$ \\
\hline Istmo & 0,0031 & 2,8682 & 16,8435 & $-19,1215$ & $-0,1447$ & 6,8896 & $1067^{*}$ & $115,73^{*}$ & $183,68^{*}$ \\
\hline Olmeca & 0,0049 & 2,6783 & 14,3412 & $-14,6723$ & $-0,1013$ & 5,6182 & $483^{*}$ & $109,28^{*}$ & $165,20^{*}$ \\
\hline \multicolumn{9}{|c|}{ Panel B: periodo de crisis 2003-2009 } & \\
\hline WTI & 0,0572 & 2,6331 & 14,4165 & $-12,8371$ & 0,0142 & 7,3952 & $1359^{*}$ & $433,43^{*}$ & $1699^{*}$ \\
\hline Brent & 0,0605 & 2,3642 & 17,9734 & $-16,7130$ & 0,0775 & 7,9342 & $1715^{*}$ & $351,16^{*}$ & $796^{*}$ \\
\hline Maya & 0,0742 & 2,8512 & 14,4138 & $-22,0324$ & $-0,1209$ & 8,6021 & $2212^{*}$ & $292,55^{*}$ & $1252^{*}$ \\
\hline Istmo & 0,0641 & 2,7491 & 15,1623 & $-22,4612$ & $-0,5151$ & 9,5812 & $3121^{*}$ & $283,63^{*}$ & $934^{*}$ \\
\hline Olmeca & 0,0598 & 2,4923 & 14,3516 & $-16,0234$ & $-0,1632$ & 7,0252 & $1148^{*}$ & $334,33^{*}$ & $1343^{*}$ \\
\hline \multicolumn{9}{|c|}{ Panel C: periodo inestable 2010-2016 } & \\
\hline WTI & $-0,0291$ & 2,1012 & 11,2928 & $-11,1342$ & 0,1646 & 6,4592 & $831^{*}$ & $277,58^{*}$ & $937^{*}$ \\
\hline Brent & $-0,0280$ & 1,8243 & 9,8962 & $-8,0835$ & 0,3105 & 6,5043 & $871^{*}$ & $181,03^{*}$ & $563^{*}$ \\
\hline Maya & $-0,0352$ & 2,0462 & 12,0562 & $-10,1546$ & 0,2924 & 7,4372 & $1378^{*}$ & $316,92^{*}$ & $1206^{*}$ \\
\hline Istmo & $-0,0286$ & 2,0762 & 11,6314 & $-10,0234$ & 0,2278 & 6,8568 & $1037^{*}$ & $268,22^{*}$ & $925^{*}$ \\
\hline Olmeca & $-0,0281$ & 1,9421 & 9,6021 & $-8,0848$ & 0,1963 & 6,1593 & $697^{*}$ & $249,26^{*}$ & $859^{*}$ \\
\hline \multicolumn{10}{|c|}{ Panel D: pruebas de raíces unitarias de Dickey-Fuller } \\
\hline & Niveles & Diferencias & & & & & & & \\
\hline WTI & $-1,10$ & $-29,65^{*}$ & & & & & & & \\
\hline Brent & $-0,46$ & $-29,22^{*}$ & & & & & & & \\
\hline Maya & $-0,54$ & $-28,62^{*}$ & & & & & & & \\
\hline Istmo & $-0,96$ & $-29,57^{*}$ & & & & & & & \\
\hline Olmeca & $-0,89$ & $-29,55^{*}$ & & & & & & & \\
\hline
\end{tabular}

Nota: * rechazo de la hipótesis nula a un nivel de $1 \%$.

Fuente: elaboración propia con datos de Bloomberg. 
Dinámica de los rendimientos de los petróleos nacionales e internacionales
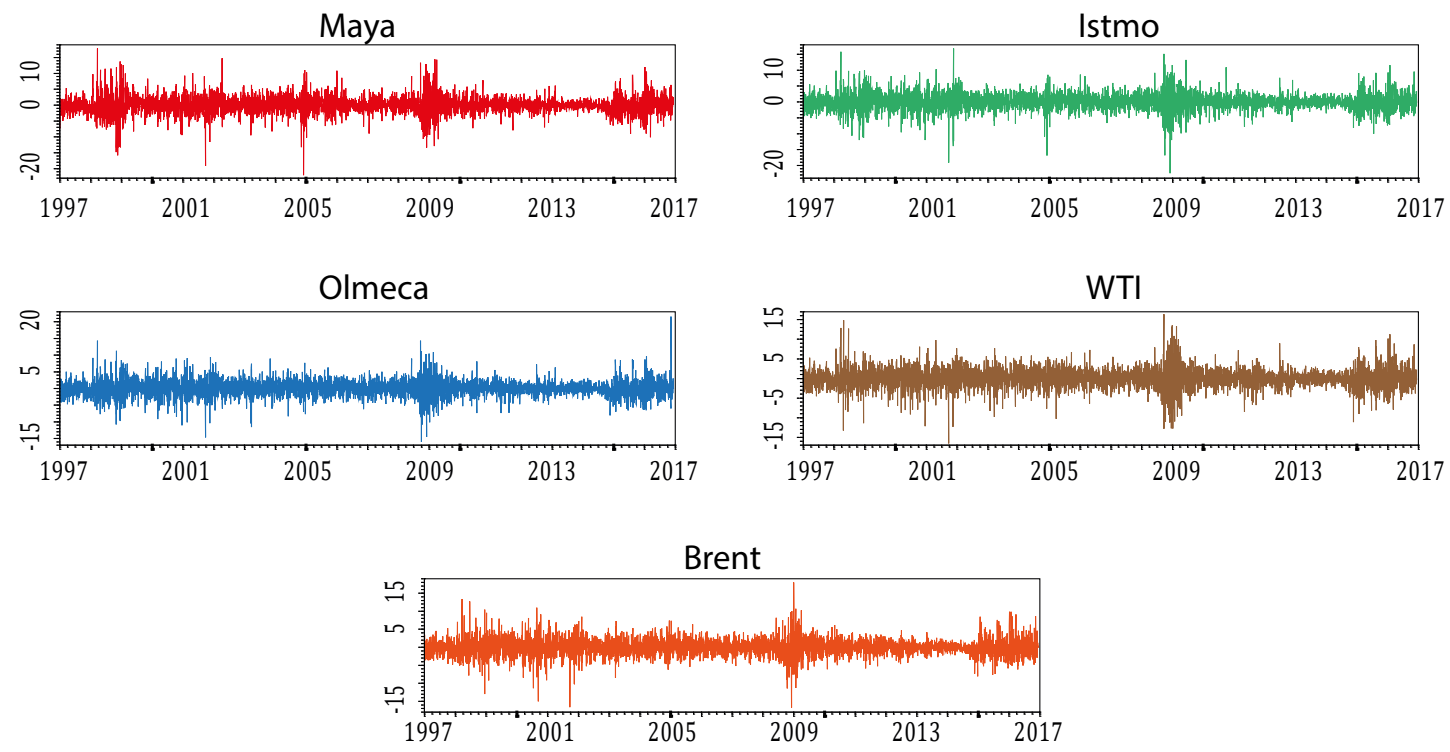

Fuente: elaboración propia con datos de Bloomberg.

diferencias son validadas con las pruebas de raíz unitaria de Dickey-Fuller aumentada, y sus resultados se reportan en el panel D de la tabla 1. Los valores negativos cercanos a cero no significativos de los estadísticos son débiles para rechazar la hipótesis nula al $1 \%$, lo cual indica que todas las series de los precios logarítmicos tienen una raíz unitaria o son integrados de orden uno, I(1). Este hallazgo puede confirmar la existencia de una relación estacionaria lineal entre dos series no estacionarias, es decir, entre los precios domésticos e internacionales. En el caso de las primeras diferencias, los grandes valores negativos rechazan la hipótesis nula de raíz unitaria, lo que confirma que las series de los rendimientos se comportan como un proceso estacionario.

La tabla 2 reporta las estimaciones de las correlaciones de Pearson, Kendall y Spearman entre los rendimientos de los precios de los petróleos internacionales y nacionales para los periodos de estabilidad, crisis e inestabilidad. Los resultados muestran que las correlaciones lineales de Pearson son significativamente positivas y más altas que las de Kendall y Spearman para cualquiera de los periodos. Pese a que los mercados internacionales y nacionales de petróleo se encuentran más integrados en la relación WTI-Olmeca, y reflejan una menor intensidad para las relaciones Brent-Maya y Brent-Olmeca, la medida de correlación de Pearson no es robusta y puede proporcionar estimaciones sesgadas con respecto al grado de integración cuando las series de los rendimientos no son estacionarias o distribuidas normalmente.

Por otra parte, aunque las correlaciones estimadas son aún positivas en los periodos de estabilidad, crisis e inestabilidad, los resultados indican que el grado de integración entre los mercados internacionales y nacionales tiende a disminuir notablemente en el periodo de estabilidad. Por ejemplo, correlaciones máximas son alcanzadas en la relación WTI-Olmeca bajo el coeficiente de Kendall y Spearman, con valores de 0,6268 y 0,7855 en el periodo de crisis 2003-2009 y con valores de 0,6503 y 0,8070 en el periodo de inestabilidad 2010-2016, contra los valores de 0,2494 y 0,3360 del periodo estable de 1997-2003, seguido por las relaciones WTI-Maya y WTI-Istmo, respectivamente. En el caso del petróleo Brent, el grado de correlación es más moderado para los diferentes periodos de estudio. 
Las relaciones Brent-Olmeca y Brent-Maya alcanzan valores de 0,4862 y 0,4985 bajo el coeficiente de Kendall y de 0,6484 y 0,6629 bajo el de Spearman en los periodos de crisis e inestabilidad, contra valores de 0,2148 y 0,1502 , y de 0,3001 y 0,2165 , respectivamente, en el periodo de estabilidad.

La fuerte variabilidad en las correlaciones es consistente con la evidencia de que en periodos altamente volátiles los mercados de petróleo parecen mantener una estructura más dependiente entre sí. Este hecho, con implicaciones negativas sobre los beneficios de la diversificación de los inversionistas participantes en los correspondientes mercados, tal vez puede ser explicado por la premisa de que los precios del petróleo han sido afectados por los precios de referencia internacional, tal como lo documenta Jiao et al. (2007), pese a que las correlaciones estimadas en el periodo de crisis difieren notablemente de las correlaciones de los periodos de estabilidad e inestabilidad. Sin embargo, los resultados no son concluyentes para sustentar estadísticamente que el mercado del petróleo mexicano está perfectamente integrado o regionalizado a los mercados internacionales, puesto que las correlaciones son sensibles a los efectos de la alta volatilidad y su persistencia. Por ello, se sugiere un análisis fuera de la muestra de la evolución de la estructura de las correlaciones a través del tiempo con modelos GARCH multivariados y pruebas estadísticas robustas, en función de probar el grado de integración entre mercados de petróleo de diferente calidad y regiones geográficas.

\section{METODOLOGÍA ECONOMÉTRICA}

\section{Especificación del modelo econométrico}

Para analizar el grado de integración entre los mercados de petróleo mexicano y de referencia internacional a través del comportamiento de la estructura de las correlaciones en los tres periodos de estabilidad, crisis e inestabilidad, este trabajo emplea el modelo de correlación condicional dinámico (GARCH-CCD) propuesto por Engle (2002) y estima las CCD entre los mercados de petróleo.

Tabla 2.

Correlaciones estimadas de los rendimientos del petróleo

\begin{tabular}{|c|c|c|c|c|c|c|}
\hline \multicolumn{4}{|c|}{ WTI } & \multicolumn{3}{|c|}{ Brent } \\
\hline \multicolumn{2}{|c|}{ Pearson } & Kendall & Spearman & Pearson & Kendall & Spearman \\
\hline \multicolumn{7}{|c|}{ Panel A: periodo estable 1997-2003 } \\
\hline Maya & 0,2768 & 0,1917 & 0,2662 & 0,2087 & 0,1502 & 0,2165 \\
\hline Istmo & 0,2901 & 0,2050 & 0,2772 & 0,2239 & 0,1641 & 0,2307 \\
\hline Olmeca & 0,3449 & 0,2494 & 0,3360 & 0,2852 & 0,2148 & 0,3001 \\
\hline \multicolumn{7}{|c|}{ Panel B: periodo de crisis 2003-2009 } \\
\hline Maya & 0,5559 & 0,3881 & 0,5110 & 0,4233 & 0,3309 & 0,4514 \\
\hline Istmo & 0,4461 & 0,3070 & 0,4073 & 0,3998 & 0,2711 & 0,3758 \\
\hline Olmeca & 0,7901 & 0,6268 & 0,7855 & 0,6183 & 0,4862 & 0,6484 \\
\hline \multicolumn{7}{|c|}{ Panel C: periodo inestable 2010-2016 } \\
\hline Maya & 0,7275 & 0,5113 & 0,6733 & 0,7060 & 0,4985 & 0,6629 \\
\hline Istmo & 0,7514 & 0,5700 & 0,7149 & 0,5906 & 0,4008 & 0,5468 \\
\hline Olmeca & 0,8290 & 0,6503 & 0,8070 & 0,6827 & 0,4816 & 0,6436 \\
\hline
\end{tabular}

Fuente: elaboración propia con datos de Bloomberg. 
Con respecto a otras alternativas ${ }^{6}$, el modelo GARCH-CCD tiene la principal ventaja: permite capturar posibles cambios de régimen en las CCD a lo largo del tiempo, esto es, respuesta de reacción a las malas noticias, innovaciones y crisis financieras. Además, el modelo relaja la carga computacional relacionada con la debilidad de la dimensión cuando el número de variables se incrementan durante la implementación numérica, e incluso permite variables explicativas adicionales en la ecuación de la media para explicar el efecto de un factor global. Una de las desventajas del GARCH-CCD es que el uso de los residuales estandarizados reduce el potencial del modelo para estimar correlaciones condicionales dinámicas y no especifica la existencia de condiciones de regularidad, momentos y propiedades asintóticas (Caporin y McAleer, 2013). Pese a ello, el modelo GARCH-CCD ha sido aceptado ampliamente en la literatura financiera para estimar y predecir las correlaciones fuera de la muestra en los mercados accionarios y de energía. Además, Laurent, Rombouts y Violante (2012) confirman el desempeño predictivo del modelo GARCH-CCD entre una familia de modelos GARCH multivariados.

El proceso de estimación de las CCD a través del modelo GARCH-CCD se desarrolla en dos etapas. De esta manera, las ecuaciones de la media condicional para los petróleos mexicanos y de referencia internacional son definidas así:

$$
\begin{aligned}
& r_{i, t}=\phi_{0}+\phi_{1} r_{i, t-1}+\phi_{2} r_{i, t-1}^{\mathrm{WTI}, \mathrm{Brent}}+\phi_{3} \varepsilon_{i, t-1}+\varepsilon_{t^{\prime}} \\
& \varepsilon_{i, t} \mid \Omega_{t-1} \rightarrow N\left(0, H_{t}\right)
\end{aligned}
$$

Donde $r_{i, t}=\left(r_{1, t}^{\text {Maya }}, r_{2, t}^{\text {Istmo }}, r_{3, t}^{\text {Olmeca }}, r_{4, t}^{\text {WTI }}, r_{5, t}^{\text {Brent }}\right)^{\prime}$ es el vector de rendimientos de los petróleos nacionales e internacionales en el tiempo $t$. La autocorrelación es corregida a través de un modelo $\operatorname{ARMA}(1,1)$, es decir, $r_{i, t-1}$ y $\varepsilon_{i, t-1}$. Los rendimientos desfasados en un periodo para los petróleos de referencia internacional, $r_{i, t-1}^{\text {WTI,Brent }}$, son utilizados para capturar el factor global y las diferencias de horarios de operación en los respectivos mercados. Este

$6 \quad$ En el contexto multivariado, la familia de modelos GARCH está integrada por el modelo VEC diagonal, el modelo BEKK y el modelo de correlación condicional constante. último solo afecta a la media de los rendimientos del petróleo mexicano porque coadyuva a confirmar el impacto de los mercados internacionales sobre los mercados del petróleo mexicano. $H_{t}$ es la matriz de varianzas-covarianzas condicionales, en tanto $\varepsilon_{i, t}=\left(\varepsilon_{1, t}^{\text {Maya }}, \varepsilon_{2, t}^{\text {Istmo }}, \varepsilon_{3, t}^{\text {Olmeca }}, \varepsilon_{4, t}^{\text {WTI }}, \varepsilon_{5, t}^{\text {Brent }}\right)^{\prime}$ es el vector del término de error estocástico o ruido blanco ${ }^{7}$.

En la primera fase se lleva a cabo la estimación de los parámetros de los modelos GARCH estándar, seguidos inmediatamente de las CCD en la segunda fase:

$H_{t}=D_{t} R_{t} D_{t}$

Donde $R_{t}$ representa la matriz simétrica de correlaciones dinámicas y $D_{t}=\operatorname{diag}\left(\sqrt{h_{1, t}}, \sqrt{h_{2, t}}\right)$ es la matriz diagonal de las desviaciones estándares condicionales, la cual se deriva de la estimación de los modelos GARCH estándar para los rendimientos del petróleo, con $\sqrt{h_{i, t}}$ para $i=1,2,3,4$ :

$h_{i, t}=\omega_{i}+\sum_{q=1}^{q_{i}} \alpha_{\dot{q}} \varepsilon_{i, t-q}^{2}+\sum_{p=1}^{p_{i}} \beta_{\dot{p}} h_{i, t-p}$

Donde $\omega_{i}$ es una constante, y $\alpha_{i}$ y $\beta_{i}$ y son los coeficientes ARCH y GARCH, respectivamente.

Ahora bien, la matriz de correlaciones condicionales se obtiene de los residuales estandarizados, los cuales son obtenidos de la estimación GARCH. De esta manera, la matriz $R_{t}$ se puede descomponer así:

$$
\begin{aligned}
& R_{t}=Q_{t}^{*-1} Q_{t} Q_{t}^{*-1} \\
& Q_{t}^{*-1}=\operatorname{diag}\left(1 / \sqrt{q_{1, t}}, 1 / \sqrt{q_{2, t}}\right)
\end{aligned}
$$

Donde $Q_{t}=\left|q_{j, t}\right|$ es una matriz simétrica positiva definida de los residuales estandarizados, $\xi_{i, t}=\frac{\varepsilon_{i, t}}{\sqrt{h_{i, t}}}$, que se puede expresar así:

$Q_{t}=\left(1-\theta_{1}-\theta_{2}\right) \bar{Q}+\theta_{1} \xi_{i, t-1} \xi_{i, t-1}^{\prime}+\theta_{2} Q_{t-1}$

$7 \quad$ El ajuste de las ecuaciones de la media bajo los modelos $\operatorname{ARMA}(2,2)$ y $\operatorname{AR}(2)$ fueron también considerados en el análisis, pero la estimación por máxima verosimilitud fue más estable a través del modelo ARMA $(1,1)$. 
Donde $\bar{Q}=E\left[\xi_{i, t} \xi_{i, t}^{\prime}\right]$ es la matriz de correlaciones no condicionadas de los residuales estandarizados, y $\xi_{i, t^{*}} \theta_{1}$ y $\theta_{2}$ son parámetros escalares que recogen los efectos de choques previos y las correlaciones condicionales dinámicas desfasadas sobre las actuales, por lo que deben ser positivos y $\theta_{1}+\theta_{2}<1$.

Finalmente, las correlaciones condicionales dinámicas en el tiempo $t$ se pueden expresar de la siguiente manera:

$\rho_{\ddot{y}, t}=\frac{\left(1-\theta_{1}-\right.}{\left[\left(1-\theta_{1}-\theta_{2}\right) \bar{q}_{i}+\theta_{1} \xi_{i, t-1} \xi_{i, t-1}^{\prime}+\right.}$

$\frac{\left.\theta_{2}\right) \bar{q}_{j}+\theta_{1} \xi_{i, t-1} \xi_{i, t-1}^{\prime}+\theta_{2} q_{j, t-1}}{\left.\theta_{2} q_{i, t-1}\right]^{1 / 2}\left[\left(1-\theta_{1}-\theta_{2}\right) \bar{q}_{j}+\theta_{1} \xi_{i, t-1} \xi_{i, t-1}^{\prime}+\theta_{2} q_{j, t}\right]^{1 / 2}}$

Donde $q_{i j}$ es el elemento de la $i$-ésima fila y $j$-ésima columna de la matriz $Q_{t}$.

Finalmente, el proceso de estimación de los parámetros y correlaciones condicionales del modelo GARCH-CCD se lleva a cabo mediante el método de cuasi-máxima verosimilitud. Por ello, bajo el supuesto de normalidad para las innovaciones de los rendimientos del petróleo, la función logarítmica de verosimilitud conjunta se puede expresar así:

$$
\begin{aligned}
& L(\theta)=-\frac{1}{2} \sum_{t=1}^{T}\left[\left(4 \log (2 \pi)+\log \left|D_{t}\right|^{2}+\varepsilon_{t}^{\prime} D_{t}^{-1} \varepsilon_{t}\right)+\right. \\
& \left.\left(\log \left|R_{t}\right|+\xi_{t}^{\prime} R_{t}^{-1} \xi_{t}-\xi_{t}^{\prime} \xi_{t}\right)\right]
\end{aligned}
$$

Donde $\theta$ es el número de observaciones y $\theta$ representa el vector de parámetros por estimar.

\section{Prueba estadística de integración o regionalización}

En esta sección se describe la prueba estadística que permite probar la hipótesis nula de que los mercados mexicanos e internacionales del petróleo están integrados. La prueba consiste en el análisis del comportamiento de las correlaciones cambiantes en el tiempo, esto es, utilizando las correlaciones condicionales dinámicas estimadas fuera de la muestra para los diferentes periodos: estable, crisis e inestable. Según Forbes y Rigobon (2002), la presencia de heterocedasticidad en la volatilidad de mercado puede ocasionar distorsiones en los coeficientes de las correlaciones y proporcionar resultados sesgados en el grado de integración. De esta manera, la prueba de integración puede mejorarse si se utilizan las correlaciones estimadas fuera de la muestra de los distintos periodos, en lugar de las correlaciones dentro de la muestra.

De acuerdo con los hallazgos de Cook (1998) y Candelon, Joëts y Tokpavi (2013), el grado de integración entre mercados de petróleo de diferente calidad y zonas geográficas de extracción tiende a descender durante periodos de movimientos de precios extremos, alimentado por los desequilibrios entre oferta y demanda, presiones inflacionarias, recesiones económicas y crisis financieras. De hecho, los movimientos atípicos hacen que los diferenciales en los precios sean más amplios entre los mercados de referencia y de baja calidad del petróleo, lo que favorece la hipótesis de regionalización. Para Weiner (1991), un alto grado de regionalización es alcanzado en el mercado internacional del petróleo cuando los efectos de choques de precios hacia precios regionales se han restringido solamente al mercado regional específico del crudo.

La prueba de integración tiene como objetivo comparar las correlaciones condicionales dinámicas fuera de la muestra en periodos de estabilidad, crisis e inestabilidad. Para ello es necesario contrastar las siguientes hipótesis nula y alternativa:

$$
\begin{aligned}
& H_{0}: \rho_{\ddot{j}}^{* \text { crisis }}=\rho_{j}^{* \text { estable }}, \quad H_{1}: \rho_{j}^{* \text { crisis }} \neq \rho_{j}^{* \text { estable }} \\
& H_{0}: \rho_{j}^{* \text { crisis }}=\rho_{j}^{* \text { inestable }}, \quad H_{1}: \rho_{j}^{* \text { crisis }} \neq \rho_{j}^{* \text { inestable }}
\end{aligned}
$$
sentan las CCD fuera de la muestra durante los periodos de estabilidad, crisis e inestabilidad, respectivamente. La hipótesis nula de que los mercados internacionales y nacionales se encuentran plenamente integrados es confirmada cuando los mercados del petróleo están altamente correlacionados y la estructura de las CCD fuera de la muestra presenta la misma intensidad, independientemente de si el mercado se encuentra en un periodo de estabilidad, crisis financiera o 
inestabilidad. En contraste, la regionalización en el mercado del petróleo es alcanzada a través de la hipótesis alternativa cuando las correlaciones del periodo de crisis son significativamente diferentes de las correlaciones de los periodos de estabilidad o inestabilidad.

Bajo la hipótesis nula, Collins y Biekpe (2003) demostraron que el estadístico de la prueba propuesta sigue una distribución $t$-Student con $\left(n^{\text {estable }}+n^{\text {crisis }}-4\right)$ o $\left(n^{\text {estable }}+n^{\text {inestable }}-4\right)$ grados de libertad, y se expresa de la siguiente manera:

$$
t=\frac{\left(\rho_{\ddot{j}}^{* \text { crisis }}-\rho_{j}^{*^{*} \text { estable }}\right)}{\sqrt{\frac{n^{\text {estable }}+n^{\text {crisis }}-4}{1-\left(\rho_{j}^{* \text { crisis }}-\rho_{j}^{\left.*^{\text {estable }}\right)}\right.}}}
$$

Donde $n^{\text {estable }}, n^{\text {crisis }}$ y $n^{\text {inestable }}$ se refieren al número de correlaciones estimadas en los periodos de estabilidad, crisis e inestabilidad ${ }^{8}$.

Para obtener resultados robustos de la prueba de integración de los mercados de petróleo, el valor de la probabilidad del estadístico $t$-Student se determina mediante el uso del método bootstrap estacionario propuesto por Politis y Romano (1994). Este procedimiento consiste en la generación de muestras bootstrap $\left(d_{b, 1}^{*}, \ldots, d_{b, n}^{*}\right)$ con $b=1, \ldots, B$. Para encontrar la distribución del estadístico bajo la hipótesis nula, el estadístico $t_{b, n}^{*}$ es calculado para cada una de las muestras bootstrap con $d$-variables, las cuales están definidas por $d_{b, n}^{*}=\rho_{i j}^{*}$ crisis $-\rho_{i j}^{* \text { estable }} \quad o$ $d_{b, n}^{*}=\rho_{i j}^{*}$ crisis $-\rho_{i j}^{* \text { inestable }}$. Para alcanzar resultados confiables y que no afecten a las muestras actuales, $B$ debe ser muy grande. De esta manera, el valor de la probabilidad bootstrap está definido por:

Valor- $p=B^{-1} \sum_{b=1}^{B} 1_{\left\{t_{b, n}^{*}>t\right\}}$

Para el nivel de significancia $\alpha$, la hipótesis nula se rechaza cuando el valor- $p$ bootstrap es menor a $\alpha$. Para determinar la significancia estadística se generan 10.000 muestras bootstrap a partir de

8 Por cuestiones de extensión, el artículo solo describe la prueba $t$ para la relación de los periodos de estabilidad y crisis; sin embargo, el estadístico para la relación de los periodos de crisis e inestabilidad también será calculado. las muestras originales de las CCD, en los diferentes periodos de análisis.

\section{EVIDENCIA EMPÍRICA DE LOS MERCADOS DE PETRÓLEO}

En esta sección se presentan los resultados de los parámetros estimados del modelo GARCH-CCD y el comportamiento de las CCD fuera de la muestra, así como los resultados de la hipótesis nula, que evidencian la existencia de integración entre los mercados del petróleo mexicano y los mercados internacionales del petróleo.

\section{Estimaciones del modelo GARCH-CCD}

En los paneles A, B, C y D de la tabla 3, los parámetros estimados del modelo GARCH-CCD son reportados para cada par de mercados, esto es, WTI y Brent contra Maya, Istmo y Olmeca, en los periodos de estabilidad y crisis ${ }^{9}$, así como las pruebas de diagnóstico para los residuales estandarizados simples y cuadrados. El coeficiente constante de la ecuación de la media condicional es pequeño y no significativo estadísticamente en la mayoría de los mercados de petróleo, excepto para el WTI en el periodo de crisis. El coeficiente del modelo AR(1), $\phi_{1}$, es significativo y positivo (negativo para el petróleo WTI) en ambos periodos, hecho que implica la presencia de persistencia en el comportamiento de los rendimientos del petróleo. El coeficiente $\phi_{2}$ del impacto de los mercados de petróleo de referencia internacional (WTI y Brent) hacia los mercados del petróleo mexicano (Maya, Istmo y Olmeca) es altamente significativo y positivo en la mayoría de los casos, con valores de 0,2421 a 0,7544 en el periodo estable y de 0,3967 a 0,5301 en el de crisis.

Los hallazgos confirman el notable control desfasado de los mercados internacionales de petróleo. No obstante, es importante resaltar que el coeficiente no es significativo en algunos mercados

9 Por falta de espacio, las estimaciones del modelo GARCHCCD no son reportados para el periodo de inestabilidad, pero los resultados se encuentran disponibles para cualquier aclaración. 
del petróleo (Maya e Istmo) durante el periodo de crisis. Finalmente, el coeficiente $\phi_{3}$ del proceso MA(1) es significativo a un nivel de $1 \%$, pero con valor mixto. Este resultado implica que el modelo MA (1) es suficiente para eliminar la autocorrelación en las series de los rendimientos, la cual es generalmente originada por la falta de sincronización en las operaciones.

De acuerdo con la ecuación de la varianza condicional, todos los coeficientes son positivos y significativos estadísticamente a un nivel de 1\%, lo que confirma que la evolución de la volatilidad de los mercados del petróleo cambia en el tiempo. Además, los coeficientes de los términos GARCH presentan valores mayores a los coeficientes de los términos ARCH, hallazgo que indica que los mercados del petróleo tienen la capacidad para autoajustarse y, a su vez, que los choques exógenos presentan una baja tasa de cambio en la volatilidad condicional. Por otra parte, la suma de los términos ARCH y GARCH es aproximadamente igual a uno para todos los mercados del petróleo estudiados. Este hecho confirma la existencia de un alto nivel de persistencia en la volatilidad, la cual varía entre 0,8792 y 0,9865 en el periodo estable y entre 0,9690 y 0,9984 en el de crisis. Por consiguiente, la varianza condicional satisface la condición de convergencia hacia su nivel de equilibrio y garantiza varianzas positivas en el proceso GARCH.

En cuanto a la ecuación de las CCD, los parámetros estimados $\theta_{1}$ y $\theta_{2}$ son estadísticamente significativos a un nivel de $1 \%$, lo que implica que la evolución de las correlaciones cambian notablemente a través del tiempo. Para el mercado de petróleo Brent, la suma de los coeficientes $\theta_{1}$ y $\theta_{2}$ es menor a 1 , con valores entre 0,9776 (estable) y 0,9919 (crisis), mientras que en el mercado del petróleo WTI alcanza valores entre 0,9803 (estable) y 0,9870 (crisis). Este hallazgo significa que las CCD exhiben un alto nivel de persistencia, particularmente en el periodo de crisis. Finalmente, $Q(p)$ y $Q^{2}(p)$ indican los resultados de la prueba de Ljung-Box para los residuales simples y cuadrados de orden $p$, esto es, desde 1 hasta orden 20 en la mayoría de los casos. La insignificancia de los estadísticos y el alto valor- $p$ confirman la ausencia de autocorrelación y heterocedasticidad en los residuales estandarizados simples y cuadrados. En consecuencia, puede afirmarse que el modelo GARCH-CCD está bien especificado y el análisis de las CCD puede llevarse a cabo sin ningún problema.

\section{Análisis de las correlaciones condicionales dinámicas}

En esta sección se analiza la dinámica de las correlaciones fuera de la muestra entre los mercados de petróleo de referencia internacional y cada uno de los mercados del petróleo mexicano durante los periodos de estabilidad, crisis e inestabilidad, que corresponden a las siguientes fechas: 04/01/2002 a $30 / 06 / 2003,01 / 07 / 2008$ a $31 / 12 / 2009$ y 02/09/2014 a 03/03/2016, respectivamente. El tamaño de la muestra total equivale a 388 estimaciones fuera de la muestra para cada uno de los periodos, los cuales fueron seleccionados de acuerdo con los eventos que agudizaron la crisis financiera global, iniciada con la absorción del banco Bear Stearns, la quiebra de Lehman Brothers y la compra de Merrill Lynch por parte de Bank of America el 17 de septiembre del 2008.

La crisis financiera global ha sido considerada por los expertos como la recesión más profunda desde la Segunda Guerra Mundial, dado que sus efectos negativos se extendieron rápidamente por todo el mundo hasta impactar a la Comunidad Europea en el 2010, principalmente a los países de Grecia, Italia, Irlanda, Portugal y España. Otro evento relevante es la sorprendente caída del $72 \%$ de los precios de los crudos en el periodo 20142016, cuando regresaron a los niveles del 2003, es decir, por debajo de los 28 dólares por barril. Esta fuerte caída ha sido impulsada por el exceso de oferta del hidrocarburo y tiene por causa principal los desacuerdos entre los miembros de la OPEP para reducir la producción de crudo, el incremento en la producción de Estados Unidos y la tensión en Medio Oriente entre Arabia Saudita e Irán. Otros importantes factores económicos se atribuyen a la 
Tabla 3.

Estimación de los parámetros del modelo GARCH-CCD

\begin{tabular}{|c|c|c|c|c|c|c|c|c|c|c|}
\hline \multirow{2}{*}{ Tipo de crudo } & \multicolumn{4}{|c|}{ Ecuación de la media condicional } & \multicolumn{6}{|c|}{ Ecuación de la varianza condicional } \\
\hline & $\phi_{0}$ & $\phi_{1}$ & $\phi_{2}$ & $\phi_{3}$ & $\omega$ & $\alpha$ & $\beta$ & $\alpha+\beta$ & $Q(p)$ & $Q^{2}(p)$ \\
\hline \multicolumn{11}{|c|}{ Panel A: periodo estable 1997-2003 } \\
\hline \multirow{2}{*}{ Brent } & 0,0102 & $0,7372^{* *}$ & & $-0,7115^{* *}$ & $0,1677^{*}$ & $0,0637^{*}$ & $0,9171^{*}$ & 0,9808 & 20,5302 & 15,3807 \\
\hline & $(0,0202)$ & $(0,3011)$ & & $(0,3131)$ & $(0,0372)$ & $(0,0064)$ & $(0,0089)$ & & {$[0,4252]$} & {$[0,7542]$} \\
\hline \multirow{2}{*}{ Maya } & 0,0132 & $0,3392^{*}$ & $0,5350^{*}$ & $-0,4963^{*}$ & $0,1454^{*}$ & $0,1336^{*}$ & $0,8489^{*}$ & 0,9825 & 26,8103 & 15,5223 \\
\hline & $(0,0219)$ & $(0,0931)$ & $(0,0217)$ & $(0,0888)$ & $(0,0263)$ & $(0,0122)$ & $(0,0370)$ & & {$[0,1407]$} & {$[0,7458]$} \\
\hline \multirow{2}{*}{ Istmo } & 0,0007 & $0,0749^{* *}$ & $0,6765^{*}$ & $-0,3930^{*}$ & $0,1381^{*}$ & $0,1255^{*}$ & $0,8520^{*}$ & 0,9775 & 8,3087 & 6,3308 \\
\hline & $(0,0207)$ & $(0,0292)$ & $(0,0172)$ & $(0,0413)$ & $(0,0282)$ & $(0,0124)$ & $(0,0127)$ & & {$[0,2163]$} & {$[0,3872]$} \\
\hline \multirow{2}{*}{ Olmeca } & 0,0011 & $0,8458^{*}$ & $0,2421^{*}$ & $-0,9242^{*}$ & $0,2308^{*}$ & $0,1223^{*}$ & $0,8244^{*}$ & 0,9467 & 20,924 & 21,7174 \\
\hline & $(0,0213)$ & $(0,0739)$ & $(0,0354)$ & $(0,0813)$ & $(0,0453)$ & $(0,0149)$ & $(0,0181)$ & & {$[0,4016]$} & {$[0,3560]$} \\
\hline \multicolumn{11}{|c|}{ Ecuación de las correlaciones condicionales dinámicas multivariadas } \\
\hline$\theta_{1}$ & $0,0681^{*}$ & $(0,0051)$ & & & & & & & & \\
\hline$\theta_{2}$ & $0,9095^{*}$ & $(0,0069)$ & & & & & & & & \\
\hline \multicolumn{11}{|c|}{ Panel B: periodo de crisis 2003-2009 } \\
\hline \multirow{2}{*}{ Brent } & 0,0437 & $0,6544^{* *}$ & & $-0,6528^{*}$ & $0,0565^{*}$ & $0,0419^{*}$ & $0,9465^{*}$ & 0,9884 & 17,1373 & 24,1973 \\
\hline & $(0,6437)$ & $(0,0512)$ & & $(0,0945)$ & $(0,0214)$ & $(0,0087)$ & $(0,0114)$ & & {$[0,6440]$} & {$[0,2339]$} \\
\hline \multirow{2}{*}{ Maya } & 0,0085 & $0,6450^{*}$ & $-0,0434$ & $-1,0121^{*}$ & $0,1403^{*}$ & $0,1399^{*}$ & $0,8462^{*}$ & 0,9841 & 24,3888 & 20,5564 \\
\hline & $(0,0058)$ & $(0,1606)$ & $(0,0968)$ & $(0,1625)$ & $(0,0149)$ & $(0,0136)$ & $(0,0038)$ & & {$[0,2258]$} & {$[0,4236]$} \\
\hline \multirow{2}{*}{ Istmo } & 0,0040 & $0,3048^{*}$ & $0,5301^{*}$ & $0,5768^{*}$ & $0,1107^{*}$ & $0,1172^{*}$ & $0,8699^{*}$ & 0,9871 & 17,1708 & \begin{tabular}{|l}
19,8788 \\
\end{tabular} \\
\hline & $(0,0171)$ & $(0,0371)$ & $(0,0600)$ & $(0,0875)$ & $(0,0196)$ & $(0,0107)$ & $(0,0112)$ & & {$[0,6419]$} & {$[0,4655]$} \\
\hline \multirow{2}{*}{ Olmeca } & 0,0184 & $0,2573^{*}$ & $0,3967^{* *}$ & 0,0318 & $0,0822^{*}$ & $0,0785^{*}$ & $0,8905^{*}$ & 0,9690 & 24,6868 & 29,9461 \\
\hline & $(0,0195)$ & $(0,0579)$ & $(0,0455)$ & $(0,2041)$ & $(0,0137)$ & $(0,0098)$ & $(0,0126)$ & & {$[0,3123]$} & {$[0,1198]$} \\
\hline \multicolumn{11}{|c|}{ Ecuación de las correlaciones condicionales dinámicas multivariadas } \\
\hline$\theta_{1}$ & $0.0475^{*}$ & $(0.0026)$ & & & & & & & & \\
\hline$\theta_{2}$ & $0.944^{4^{*}}$ & $(0.0028)$ & & & & & & & & \\
\hline \multicolumn{11}{|c|}{ Panel C: periodo estable 1997-2003 } \\
\hline \multirow{2}{*}{ WTI } & 0,0243 & $-0,7157^{*}$ & & $0,7754^{*}$ & $0,7989^{*}$ & $0,1135^{*}$ & $0,7657^{*}$ & 0,8792 & 24,6868 & 29,9461 \\
\hline & $(0,0278)$ & $(0,1290)$ & & $(0,1149)$ & $(0,1812)$ & $(0,0144)$ & $(0,0374)$ & & {$[0,3123]$} & {$[0,1198]$} \\
\hline \multirow{2}{*}{ Maya } & 0,0263 & $0,2248^{* *}$ & $0,6393^{*}$ & $-0,4274^{*}$ & $0,1028^{*}$ & 0,1109* & $0,8753^{*}$ & 0,9862 & 19,3615 & 22,9122 \\
\hline & $(0,0248)$ & $(0,0099)$ & $(0,0213)$ & $(0,1039)$ & $(0,0167)$ & $(0,0093)$ & $(0,0096)$ & & {$[0,4985]$} & {$[0,2931]$} \\
\hline \multirow{2}{*}{ Istmo } & 0,0100 & $0,2111^{*}$ & $0,7544^{*}$ & $-0,6051^{*}$ & $0,1981^{*}$ & $0,2214^{*}$ & $0,7381^{*}$ & 0,9895 & 30,6846 & \begin{tabular}{|l|}
16,2188 \\
\end{tabular} \\
\hline & $(0,0208)$ & $(0,0626)$ & $(0,0161)$ & $(0,0489)$ & $(0,0263)$ & $(0,0209)$ & $(0,0163)$ & & {$[0,1002]$} & {$[0,8004]$} \\
\hline & 0,0151 & $0,2598^{*}$ & $0,4760^{*}$ & $-0,6974^{*}$ & $0,1498^{*}$ & $0,0925^{*}$ & $0,8719^{*}$ & 0,9644 & 14,3477 & 17,7901 \\
\hline Olmeca & $(0,0130)$ & $(0,0489)$ & $(0,0228)$ & $(0,0366)$ & $(0,0252)$ & $(0,0113)$ & $(0,0130)$ & & {$[0,8124]$} & {$[0,6012]$} \\
\hline & & cuación d & s correla & es condici & les dinámi & as multiv & ariadas & & & \\
\hline$\theta_{1}$ & $0,0853^{*}$ & $(0,0043)$ & & & & & & & & \\
\hline$\theta_{2}$ & $0,8950^{*}$ & $(0,0051)$ & & & & & & & & \\
\hline & & & Panel D & riodo de c & $2003-200$ & & & & & \\
\hline WT & $0,2346^{* *}$ & $-0,8658^{*}$ & & $0,8423^{*}$ & $0,1064^{*}$ & $0,0643^{*}$ & $0,9170^{*}$ & 0,9813 & 14,3472 & 19,1584 \\
\hline WT & $(0,0951)$ & $(0,1338)$ & & $(0,1449)$ & $(0,0324)$ & $(0,0053)$ & $(0,0051)$ & & {$[0,8125]$} & {$[0,5116]$} \\
\hline & $-0,0052$ & $0,3799^{* *}$ & $-0,0396$ & $-0,7709^{*}$ & $0,0415^{*}$ & $0,1357^{*}$ & $0,8627^{*}$ & 0,9984 & 24,4368 & 15,0019 \\
\hline Maya & $(0,0056)$ & $(0,1273)$ & $(0,1011)$ & $(0,0948)$ & $(0,0063)$ & $(0,0127)$ & $(0,0088)$ & & {$[0,2238]$} & {$[0,7763]$} \\
\hline Istmo & $-0,0031$ & $0,7743^{*}$ & $-0,0667$ & $-1,0234^{*}$ & $0,0893^{*}$ & $0,0990^{*}$ & $0,8911^{*}$ & 0,9901 & 7,9336 & 11,2339 \\
\hline Istmo & $(0,0054)$ & $(0,1273)$ & $(0,0974)$ & $(0,0948)$ & $(0,0164)$ & $(0,0103)$ & $(0,0123)$ & & {$[0,8928]$} & {$[0,6676]$} \\
\hline & $-0,0011$ & $0,7377^{*}$ & $-0,5197^{*}$ & $-1,1351^{*}$ & $0,2962^{*}$ & $0,2507^{*}$ & $0,7196^{*}$ & 0,9703 & 15,1851 & 10,1950 \\
\hline Oimeca & $(0,0096)$ & $(0,0821)$ & $(0,0675)$ & $(0,0760)$ & $(0,0287)$ & $(0,0208)$ & $(0,0041)$ & & {$[0,3656]$} & {$[0,7478]$} \\
\hline & & cuación d & s correla & es condici & les dinámi & as multiv & ariadas & & & \\
\hline$\theta_{1}$ & $0,0691^{*}$ & $(0,0040)$ & & & & & & & & \\
\hline$\theta_{2}$ & $0,9179^{*}$ & $(0,0044)$ & & & & & & & & \\
\hline
\end{tabular}

Nota: la tabla reporta las estimaciones del modelo GARCH-CCD. ${ }^{*} \mathrm{y}^{* *}$ indican significancia a un nivel de $1 \%$ y $5 \%$, respectivamente. Los errores estándar son reportados entre paréntesis. $Q(p)$ y $Q^{2}(p)$ indican los estadísticos de la prueba de Ljung-Box para los residuales estandarizados simples y cuadrados, y los valores- $p$ aparecen entre corchetes cuadrados.

Fuente: elaboración propia con datos de Bloomberg. 
desaceleración de la economía de China y el encarecimiento del dólar estadounidense frente a otras divisas duras.

La figura 3 describe el comportamiento dinámico de los coeficientes de correlación entre los mercados nacionales e internacionales de petróleo para los diferentes periodos en estudio. Al analizar la estructura de las CCD, se puede observar un comportamiento similar al de un proceso no estacionario para cada par de series a través del tiempo; es decir, regímenes de volatilidad baja y alta para las CCD, ocasionados por el alto grado de persistencia. De ahí la importancia de tomar en cuenta el factor global alimentado por el efecto de los rendimientos de los precios de referencia hacia los rendimientos de los precios nacionales del petróleo. Durante el periodo estable se puede observar que las CCD comienzan a descender dramáticamente de 0,5201 a 0,1250 para la relación WTI-Maya, de 0,7931 a 0,2554 para la relación WTI-Istmo y de 0,6838 a 0,2196 para la relación WTI-Olmeca. Las CCD entre los rendimientos del Brent y el petróleo mexicano presentan este mismo comportamiento, pero su caída es relativamente menor debido a que es más estrecha la diferencia entre el valor máximo y mínimo.

En la fase de la crisis financiera global, la variabilidad en el nivel de las CCD es significativamente notable comparado con la fase de estabilidad, como se puede observar en las seis relaciones. No obstante, el diferencial entre el valor mínimo y máximo se reduce, al oscilar en una banda más estrecha entre valores de 0,7171 a 0,7922 para la WTI-Maya, de 0,7726 a 0,8277 para la WTI-Istmo y de 0,5061 a 0,6359 para la WTI-Olmeca, mientras que las bandas de oscilación para el Brent

Comportamiento de las correlaciones condicionales dinámicas
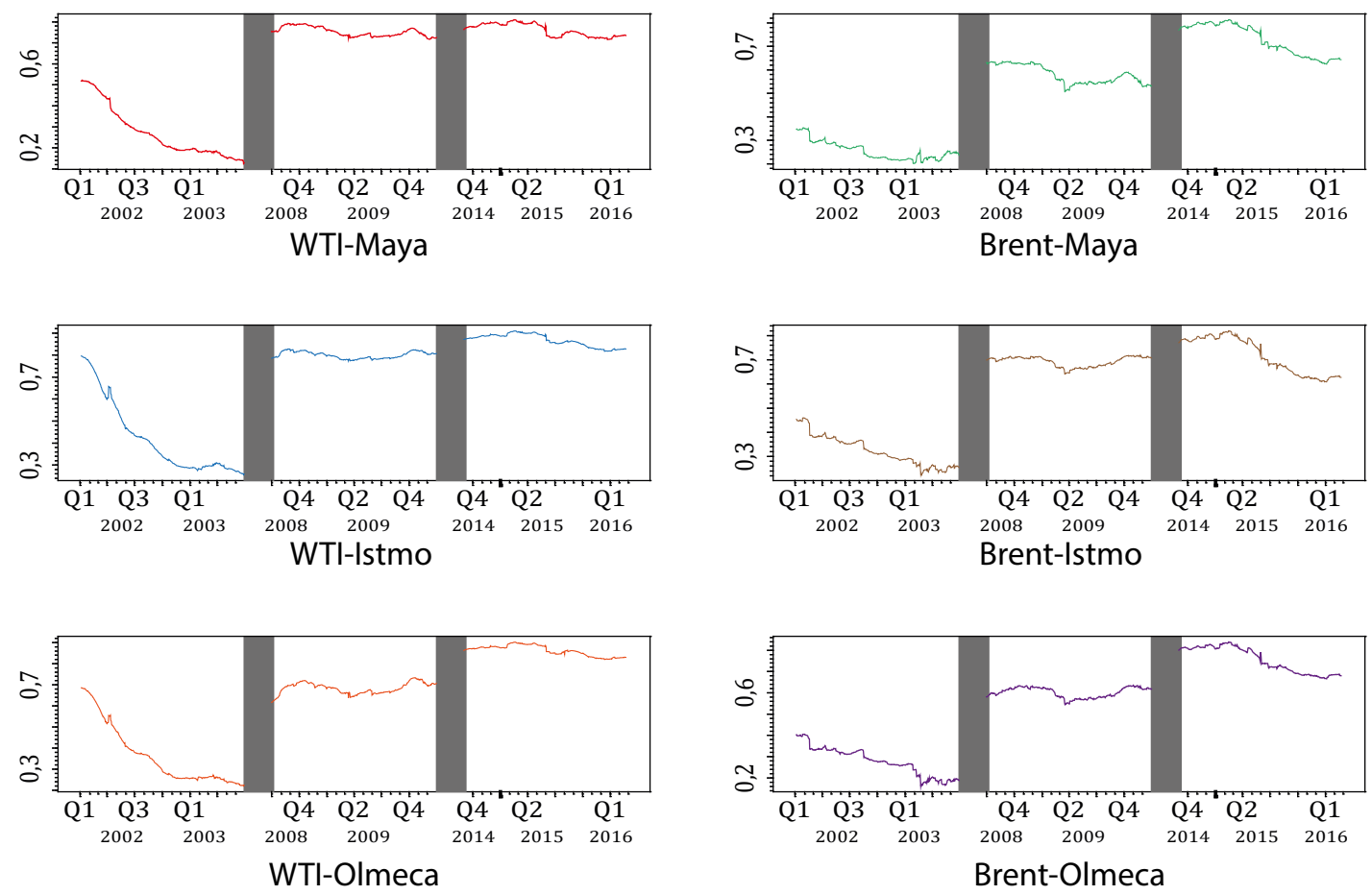

Nota: el comportamiento dinámico de las correlaciones para los diferentes periodos de análisis es separado por las barras grises.

Fuente: elaboración propia con datos de Bloomberg. 
se encuentran entre 0,5061 y 0,6359, 0,6408 y 0,7189 y 0,5449 y 0,6349 , en el mismo orden. Este periodo de análisis se caracteriza por un mayor grado de integración entre los mercados de petróleo de referencia internacional y los mercados del petróleo mexicano debido a que las correlaciones se incrementaron notablemente por el descenso en forma exponencial de los precios del petróleo, especialmente con respecto al WTI.

En el periodo de inestabilidad, las seis gráficas muestran que las CCD presentan una tendencia al alza durante los primeros nueve meses. Esta tendencia creciente se puede observar con respecto a las correlaciones del periodo estable, particularmente en las relaciones WTI-Istmo y WTI-Olmeca, que alcanzaron niveles por arriba de 0,90. Sin embargo, el patrón de las correlaciones cambia de tendencia a partir de junio del 2015, pero nunca vuelven a registrar los niveles mínimos del periodo de la crisis financiera global, excepto para la relación Brent-Istmo. El hallazgo en mención revela que las CCD reaccionan rápidamente a las condiciones cambiantes del mercado cuando los precios del petróleo presentan fuertes y prolongadas caídas. Asimismo, estos resultados ponen de manifiesto que los mercados del petróleo están altamente correlacionados y que su dinámica presenta casi la misma intensidad en los periodos de crisis e inestabilidad. Y aunque el resultado es un buen síntoma de que los mercados del petróleo están plenamente integrados en la actualidad, la evidencia no tiene un sustento estadístico.

\section{Evidencia de la prueba de integración o regionalización}

En esta sección se prueba la existencia de integración entre los mercados de petróleo de referencia internacional y el mercado de petróleo mexicano, con base en el estadístico-t. Esta prueba estadística indica que la hipótesis nula de que las correlaciones se mantienen significativamente altas y con la misma intensidad durante el periodo de crisis con respecto a las correlaciones de los periodos de estabilidad e inestabilidad. Para una validación estadística más robusta, el valor- $p$ bootstrap de la prueba de integración se estima generando 10.000 replicaciones bootstrap estacionarias para cada una de las correlaciones de los periodos.

En la tabla 4 se reportan el nivel promedio y varianza de las correlaciones estimadas fuera de la muestra, el estadístico- $t$ promedio de las 10.000 réplicas bootstrap y el valor- $p$ de la prueba de integración para cada par de rendimientos de los mercados del petróleo mexicano y de referencia internacional. Los resultados muestran que el nivel promedio de las correlaciones es significativamente positivo en los periodos 2002-2009 y 2008-2016. Con respecto al periodo estable, el nivel promedio de las correlaciones es significativamente más alto durante los periodos de crisis e inestabilidad. La tendencia creciente se mantiene ligeramente por arriba de las CCD del periodo de crisis y su intensidad es más pronunciada. Este hecho revela una relación más fuerte y estable entre los mercados nacionales e internacionales del petróleo porque los precios del crudo mexicano han experimentado comovimientos más fuertes desde el 2006 y después del 2008, lo que implica que el mercado nacional ha tenido una reacción similar a la de los mercados internacionales de petróleo ante la presencia de choques macroeconómicos externos. Los hallazgos se encuentran en línea con los resultados de Jiao et al. (2007) y Ji y Fan (2016).

En términos porcentuales, las relaciones WTI-Maya, Brent-Maya, Brent-Olmeca y BrentIstmo parecen estar más correlacionadas durante la crisis financiera global, al registrar incrementos aproximadamente del 180\%, 128\%, 117\% y 115\%, seguidos por la relación de WTI-Istmo y WTIOlmeca, con incrementos del $92 \%$ y $90 \%$, respectivamente. Tal hallazgo es alimentado por el nivel de la varianza de las correlaciones experimentado en el periodo estable, que es relativamente mayor con respecto al de crisis. Este hecho también está asociado al amplio diferencial observado entre el valor mínimo y máximo en el periodo estable, lo que indica que las correlaciones estimadas fuera de la muestra son más volátiles. 
Asimismo, las CCD del periodo inestable presentan casi la misma intensidad con respecto a las CCD del periodo de crisis, pero con incrementos relativamente más pequeños que se encuentran por debajo del $26 \%$ en promedio; incluso valores del $1,14 \%$ y $3,75 \%$ son alcanzados por las relaciones WTI-Maya y Brent-Istmo. Los resultados del nivel promedio y varianza de las correlaciones revelan que las CCD del periodo inestable tienden a fluctuar más frecuentemente en el tiempo, pero en bandas relativamente más estrechas, hallazgo que precisamente revela que la hipótesis de integración de los mercados del petróleo se fortalece más en el periodo de crisis, pues las relaciones son más estables.

Con respecto a los resultados de la prueba de integración, los valores altos y positivos del estadístico- $t$ indican el rechazo de la hipótesis nula de que las CCD estimadas fuera de la muestra de los periodos de estabilidad e inestabilidad son significativamente consistentes a las CCD del periodo de crisis, lo que significa que la mayoría de los mercados nacionales están regionalizados a los mercados internacionales de petróleo a un nivel de $5 \%$, particularmente en el periodo de comparación crisis-estable. Estos resultados son sustentados por el pequeño valor- $p$ de la distribución bootstrap, que rechaza la hipótesis de integración entre mercados internacionales y nacionales de petróleo, excepto para las relaciones Brent-Istmo y WTI-Mayal, a un nivel de significancia de $1 \%$ para los periodos de crisis e inestabilidad. La principal razón de este hallazgo se puede atribuir, por un lado, a que la especulación y los eventos geopolíticos causan un mayor nivel de ruido durante episodios de movimientos extremos y con ello fortalecen las relaciones entre los mercados nacionales e internacionales de petróleo en los periodos de crisis e inestabilidad. Por otro lado, choques de una región pueden afectar más a los precios del petróleo mexicano que a los de referencia internacional, generando diferenciales de precios más amplios y correlaciones más débiles que llevan a debilitar la relación dinámica entre los precios internacionales y domésticos y, en consecuencia, a reducir la transmisión de volatilidad de precios de los mercados internacionales hacia el mercado del petróleo mexicano.

Finalmente, el grado de regionalización observado en los mercados de petróleo durante el análisis de los periodos de crisis-estable y crisisinestable puede ser adjudicado, en general, a la falta de un sistema de fijación de precios propio y a la política energética que adopta el gobierno mexicano, al permitir que los precios del petróleo nacional sigan los movimientos de los mercados

Tabla 4.

Resultados de la prueba estadística de integración entre mercados de petróleo

\begin{tabular}{|c|c|c|c|c|c|c|c|c|c|c|}
\hline \multirow{2}{*}{ Tipo de petróleo } & \multicolumn{3}{|c|}{ Promedio } & \multicolumn{3}{|c|}{ Varianza } & \multicolumn{2}{|c|}{ Estable-crisis } & \multicolumn{2}{|c|}{ Crisis-inestable } \\
\hline & Estable & Crisis & Inestable & Estable & Crisis & Inestable & Estadístico- $t$ & Valor-p & Estadístico- $t$ & Valor-p \\
\hline \multicolumn{11}{|c|}{ Panel A } \\
\hline Brent-Maya & $0,2542^{*}$ & $0,5791^{*}$ & $0,7291^{*}$ & 0,0015 & 0,0011 & 0,0042 & $115,1008^{*}$ & 0,0008 & $38,2714^{*}$ & 0,0023 \\
\hline Brent-Istmo & $0,3223^{*}$ & $0,6925^{*}$ & $0,7185^{*}$ & 0,0037 & 0,0004 & 0,0055 & $111,1486^{*}$ & 0,0006 & $6,6322^{*}$ & 0,0392 \\
\hline Brent-Olmeca & $0,2771^{*}$ & $0,6018^{*}$ & $0,7556^{*}$ & 0,0042 & 0,0006 & 0,0036 & $90,1167^{*}$ & 0,0009 & $46,9146^{*}$ & 0,0016 \\
\hline \multicolumn{11}{|c|}{ Panel B } \\
\hline WTI-Maya & $0,2686^{*}$ & $0,7528^{*}$ & $0,7618^{*}$ & 0,0132 & 0,0005 & 0,0009 & $79,8759^{*}$ & 0,0009 & $4,3714^{*}$ & 0,0259 \\
\hline WTI-Istmo & $0,4157^{*}$ & $0,8001^{*}$ & $0,8675^{*}$ & 0,0251 & 0,0002 & 0,0008 & $46,1499^{*}$ & 0,0017 & $40,9134^{*}$ & 0,0010 \\
\hline WTI-Olmeca & $0,3608^{*}$ & $0,6850^{*}$ & $0,8625^{*}$ & 0,0184 & 0,0006 & 0,0006 & $65,5549^{*}$ & 0,0036 & $37,4336^{*}$ & 0,0062 \\
\hline
\end{tabular}

Nota: la tabla reporta el nivel promedio y varianza de las correlaciones, el estadístico- $t$ y valor- $p$ para los diferentes periodos de análisis. *indica significancia a un nivel de $5 \%$.

Fuente: elaboración propia con datos de Bloomberg. 
internacionales, a pesar de que el país sea uno de los principales productores y exportadores de crudo en todo el mundo. Más específicamente, los petróleos mexicanos mantienen características de tomadores de precios porque en su proceso de fijación dependen en gran medida de los marcadores WTI y Brent; una desventaja que genera desfases temporales en la evolución de los precios del petróleo nacional. Los hallazgos son consistentes con la evidencia empírica obtenida en la investigación de Liu, Chen y Wan (2013).

\section{CONCLUSIONES}

Este artículo analiza la hipótesis de integración o regionalización de México en los mercados internacionales de petróleo desde la perspectiva de la evolución de las correlaciones fuera de la muestra a través de una venta móvil. Para el análisis se estima un modelo GARCH-DCC que utiliza los precios de los principales marcadores internacionales de petróleo WTI y Brent y los precios de los petróleos nacionales Maya, Istmo y Olmeca durante el periodo 1997-2016. Los resultados revelan que las CCD son significativamente positivas y cambian en respuesta al origen de choques en los precios del petróleo, ya sea en periodos de relativa calma, crisis o turbulencia financiera. En general, las CCD tienden a disminuir cuando los precios en los mercados de petróleo presentan una tendencia alcista, y viceversa, y de este modo se impulsa la eficiencia de las estrategias de diversificación orientadas a limitar el impacto de los choques del petróleo, en particular para las combinaciones WTI contra Maya, Istmo y Olmeca.

Además, la presencia de movimientos extremos en los precios del petróleo incrementa el grado de correlación en los periodos de crisis e inestabilidad, lo que parece fortalecer la hipótesis de integración entre los mercados del petróleo e intensifica la presencia del riesgo sistemático a lo largo del tiempo. Sin embargo, los resultados del estadístico- $t$ y los valores- $p$ del análisis bootstrap muestran que las CCD son significativamente diferentes en los periodos de relativa tranquilidad e inestabilidad financiera con respecto a las CCD del periodo de crisis. Este resultado proporciona robusta evidencia estadística de que los mercados domésticos de petróleo se encuentran regionalizados debido a que sus precios están cada vez más influenciados por los precios internacionales del petróleo; por ello, la política energética de México no representa una fuente de volatilidad para los precios de los mercados globales de petróleo.

Los hallazgos tienen importantes implicaciones económicas y financieras para los participantes en los mercados de petróleo, en particular para el Gobierno Mexicano. La regionalización del mercado del petróleo mexicano en los mercados internacionales sugiere la implementación de un mercado de futuros que coadyuve no solo como mecanismo en el proceso de la formación de precios, sino también en el desarrollo de estrategias óptimas de cobertura para reducir la exposición al riesgo de precios cuando la hipótesis de integración entre los mercados de petróleo se fortalezca a través del tiempo y cuando la relación sea más estable en periodos de crisis o turbulencia financiera. Otra de las implicaciones de las características de integración o regionalización es que agilizará la apertura de la industria del petróleo a las inversiones nacionales y transfronterizas privadas, lo que permitirá tener un mercado de petróleo más eficiente y, en consecuencia, finanzas públicas más sanas que conduzcan al rápido crecimiento económico.

Sin embargo, la regionalización puede llegar a tener un efecto negativo en la tendencia de los precios de los hidrocarburos fósiles de la región. De hecho, la reversión de precios se reflejará inmediatamente en el financiamiento de la política gubernamental, con marcados recortes en el presupuesto del Estado para el gasto público y la inversión en infraestructura, lo que estancará el crecimiento de la economía y desarrollo del país. Finalmente, este análisis deja abierta una agenda de trabajo para capturar las características de no-linealidad y los efectos de colas anchas en las series del petróleo en diferentes escalas de tiempo a través del análisis de ondoletas (wavelets), que generalmente omiten el modelo GARCH-DCC, incluso la metodología basada en cópulas. 


\section{REFERENCIAS}

1. Adelman, M. A. (1984). International oil agreements. Energy Journal, 5(3), 1-9. https://www.jstor.org/ stable/41321691

2. Bachmeier, L. J. y Griffin, J. M. (2006). Testing for market integration crude oil, coal, and natural gas. Energy Journal, 27(2), 55-71. https://econpapers.repec.org/article/aenjournl/2006v27-02-a04.htm

3. Bentzen, J. (2007). Does OPEC influence crude oil price? Testing for co-movements and causality between regional crude oil prices. Applied Economics, 39(11), 1375-1385. https://doi.org/10.1080/00036840600606344

4. British Petroleum (2017). Statistical Review of World Energy. Londres: BP:

5. Candelon, B., Joëts, M. y Tokpavi, S. (2013). Testing for Granger causality in distribution tails: An application to oil markets integration. Economic Modeling, 31, 276-285. https://doi.org/10.1016/j. econmod.2012.11.049

6. Caporin, M. y McAleer, M. (2013). Ten things you should know about the dynamic conditional correlation representation. Econometrics, 1(1), 115-126. https://doi.org/10.3390/econometrics1010115

7. Collins, D. y Biekpe, N. (2003). Contagion a fear for Africa equity market? Journal of Economics and Business, 55(3), 285-297. https://doi.org/10.1016/S0148-6195(03)00020-1

8. Cook, J. (1998). California crude oil [Mimeo]. Recuperado de www.eia.doe.gov/pub/

9. De Jesús, R. (2016). Estrategias dinámicas de cobertura cruzada eficiente para el mercado del petróleo mexicano: evidencia de dos modelos GARCH multivariados con término de corrección de error. Economía: Teoría y Práctica, 44, 115-146. https://doi.org/10.24275/ETYPUAM/NE

10. Domínguez, R. M., Venegas, F. y Palafox, A. O. (2018). Short-and long-term relations among prices of the Mexican Crude Oil Blend, West Texas Intermediate, and Brent: Market Trend and Risk Premia, 2005-2016. International Journal of Energy Economics and Policy, 8(3), 87-91. https://ideas.repec.org/a/ eco/journ2/2018-03-13.html

11. Energy Information Administration (2012). International Energy Statistics. Recuperado de https://www. eia.gov/international/data/world

12. Engle, R. F. (2002). Dynamic conditional correlation: A simple class of multivariate generalized autoregressive conditional heteroskedasticity models. Journal of Business and Economics Statistics, 20(3), 339-350. https://doi.org/10.1198/073500102288618487

13. Ewing, B. T. y Harter, C. L. (2000). Co-movements of Alaska North Slope and UK Brent crude oil prices. Applied Economics Letters, 7(8), 553-558. https://doi.org/10.1080/13504850050033373

14. Fattouh, B. (2010). The dynamics of crude oil price differentials. Energy Economics, 32 (2), 334-342. https:// doi.org/10.1016/j.eneco.2009.06.007

15. Forbes, K. y Rigobon, R. (2002). No contagion, only interdenpendence: measuring stock market comovements. Journal of Finance, 57(5), 2223-2261. https://doi.org/10.1111/0022-1082.00494

16. Gülen, S. G. (1997). Regionalization in the world crude oil market. Energy Journal, 18(2), 109-127.

17. Gülen, S. G. (1999). Regionalization in the world crude oil market: Further results. Energy Journal, 20(1), 125-139. https://researchers.dellmed.utexas.edu/en/publications/regionalization-in-the-world -crude-oil-market-further-evidence

18. Hammoudeh, S., Thompson, M. y Ewing, B. (2008). Threshold cointegration analysis of crude oil benchmarks. Energy Journal, 29(4), 79-95. https://doi.org/10.2307/41323182 
19. Jia, X., An, H., Fang, W. Sun, X. y Huang, X. (2015). How do correlations of crude oil prices co-move? A grey correlation-based wavelet perspective. Energy Economics, 49, 588-598. https://doi.org/10.1016/j. eneco.2015.03.008

20. Jiao, J. L., Fan, Y., Wei, Y. M., Han, Z. Y. y Zhang, J. T. (2007). Analysis of the co-movements between Chinese and International crude oil price. International Journal of Global Energy, 27(1), 61-76.

21. Ji, Q. y Fan, Y. (2015). Dynamic integration of world oil prices: A reinvestigation of globalisation vs. regionalization. Applied Energy, 155(1), 171-180. https://www.deepdyve.com/lp/elsevier/ dynamic-integration-of-world-oil-prices-a-reinvestigation-of-Daf3SxZWrD

22. Kleit, A.N. (2001). Are regional oil markets growing closer together? An arbitrage cost approach. Energy Journal, 22(2), 1-15. https://doi.org/10.5547/ISSN0195-6574-EJ-Vol22-No2-1

23. Kuck, K. y Schweikert, K. (2017). A Markov regime-switching model of crude oil market integration. Journal of Commodity Markets, 6, 16-31. https://doi.org/10.1016/j.jcomm.2017.03.001

24. Lanza, A., Manera, M. y McAleer, M. (2006). Modeling dynamic conditional correlations in WTI oil forward and futures returns. Finance Research Letters, 3(2), 114-132. https://doi.org/10.1016/j.frl.2006.01.005

25. Laurent, S., Rombouts, J. V. y Violante, F. (2012). On the forecasting accuracy of multivariate GARCH models. Journal of Applied Econometrics, 27(6), 934-955. https://doi.org/10.1002/jae.1248

26. Li, R. y Leung, G. C. (2011). The integration of China into the world crude oil market since 1998. Energy Policy, 39(9), 5159-5165. https://ideas.repec.org/a/eee/enepol/v39y2011i9p5159-5166.html

27. Liao, H. C., Lin, S. C. y Huang, H. C. (2014). Are crude oil markets globalized or regionalized? Evidence from WTI and Brent. Applied Economics Letters, 21 (4), 235-241.

28. Liu, L., Chen, C. y Wan, J. (2013). Is world oil market "one great pool"? An example from China's and international oil market. Economic Modelling, 35, 364-373. https://doi.org/10.1016/j.econmod.2013.07.027

29. Lu, F., Hong, Y., Wang, S. Lai, K. y Liu, J. (2014). Time-varying Granger causality tests for applications in global crude oil markets. Energy Economics, 42, 289-298. https://doi.org/10.1016/j.eneco.2014.01.002

30. Milonas, N. y Henker, T. (2001). Price spread and convenience yield behaviour in the international oil market. Applied Financial Economics, 11(1), 23-36. https://doi.org/10.1080/09603100150210237

31. Montepeque, J. (2005). Sour crude pricing: A pressing global issue. Middle East Economic Survey, 48(14), $1-42$.

32. Politis, D. N. y Romano, J. P. (1994). The stationary bootstrap. Journal of the American Statistical Association, 89(428), 1303-1313. https://doi.org/10.1080/01621459.1994.10476870

33. Reboredo, J. C. (2011). How do crude oil prices co-move? A copula approach. Energy Economics, 33(5), 948-955. https://ideas.repec.org/a/eee/eneeco/v33y2011i5p948-955.html

34. Ruiz, A. y Anguiano, J. E. (2016). Modelación de las dinámicas, volatilidades e interrelaciones de los rendimientos del petróleo mexicano, BRENT y WTI. Ensayos, Revista de Economía, 2, 175-194. https:// ideas.repec.org/a/ere/journl/vxxxvy2016i2p175-194.html

35. Weiner, R. J. (1991). Is the world oil market one great pool? Energy Journal, 12(3), 95-107. https://pdfs. semanticscholar.org/cf53/f3cd19d2dfc859ada89d740c4910c6fe333e.pdf

36. Wilmot, N. A. (2013). Cointegration in the oil market among regional blends. International Journal Energy Economic Policy, 3(4), 424-433. https://experts.umn.edu/en/publications/cointegration-in-the-oil-market -among-regional-blends 